\title{
Clinical Application of a Microbiological Study on Chronic Suppurative Otitis Media
}

\author{
Prayaga N. Srinivas Moorthy ${ }^{1}$, Jadi Lingaiah ${ }^{2}$, Sudhakar Katari ${ }^{2}$, Anil Nakirakanti ${ }^{2}$ \\ ${ }^{1}$ Department of E.N.T.-Head \& Neck Surgery, Deccan College of Medical Sciences, Hyderabad, India \\ ${ }^{2}$ Department of E.N.T.-Head \& Neck Surgery, Chalmeda Anand Rao Institute of Medical Sciences, Karimnagar, India \\ Email: pns_moorthy@yahoo.com
}

Received March 18, 2013; revised April 19, 2013; accepted May 15, 2013

Copyright (c) 2013 Prayaga N. Srinivas Moorthy et al. This is an open access article distributed under the Creative Commons Attribution License, which permits unrestricted use, distribution, and reproduction in any medium, provided the original work is properly cited.

\begin{abstract}
Our prospective study conducted over a period of 9 months includes 100 samples of ear discharge collected from 90 patients suffering from tubo-tympanic type of chronic suppurative otitis media (CSOM). The ear discharge which is collected with sterile swabs is subjected to Gram's staining and culture of the causative organism. Antibiotic sensitivity test of cultured bacterial growth is undertaken to know the susceptibility of the causative organism. Of the 100 samples, 62 were culture positive where Pseudomonas aeruginosa was the most common pathogen followed by Staphylococcus aureus. Most of the cultured organisms in our study were sensitive to drug Ciprofloxacin. The outcome of our study enabled us to set an empirical medical treatment for an early resolution of ear discharge and inflammation in our patients with CSOM as we could understand the aetiological pathogens and their susceptibility pattern. Effective medical treatment in obtaining a discharge free ear prior to surgical treatment led us to improve the surgical outcome in our patients with CSOM.
\end{abstract}

Keywords: Chronic Suppurative Otitis Media; Microbiology; Antibiotic Resistance; Ciprofloxacin

\section{Introduction}

Otitis media, an inflammation of the middle ear, is quite common in the developing world [1]. Based on the duration of symptoms, like ear discharge or perforation of the tympanic membrane, it is labeled as chronic otitis media when the symptoms are present beyond 12 weeks. Chronic suppurative otitis media (CSOM) is inflammation of the middle ear cleft and its contents which are classically divided into 1) a tubo-tympanic type affecting the middle ear mucoperiosteum and 2) an attico-antral type which is an active squamous disease within a growth of squamous epithelium into the middle ear cleft. CSOM is the leading cause of preventable hearing loss in the developing world [1]. Our institute, Chalmeda Anandrao Institute of Medical Sciences (CAIMS), is located in Karimnagar district of India and caters to the health needs of rural population of adjoining four districts. Patients with a tubotympanic type of CSOM constitute a major proportion of patients who belong to low socioeconomic status and have inadequate health education. In effect, they present it to us late and are often with inade- quate and improper treatment. The aetiology of a tubotympanic type of CSOM involves ascending infection from nasopharynx through the Eustachian tube or an infection entering from external auditory canal through a perforation in tympanic membrane. The aim of our study on bacterial aetiology and their antibiotic susceptibility pattern is to know whether an empirical treatment based on the result of the study will effectively shorten the period of infection associated with discharge in patients of CSOM and thereby improve the surgical outcome.

\section{Materials and Methods}

The present study is a prospective study involving 90 patients examined at CAIMS and 100 samples of ear discharge were collected from these patients. Out of 90 patients 10 patients had bilateral active ear discharge and swabs were collected from both the ears separately.

The inclusion criteria for the study are 1) a patient with active ear discharge who has not used any antibiotic medication either topical or systemic for at least 7 days immediately preceding to presentation to us, irrespective 
of age and sex 2) only patients with tubo-tympanic type of CSOM with a central perforation are included. A thorough history with regard to duration of illness, associated symptoms and usage of antibiotics has been taken prior to inclusion in the study.

The clinical features of patients included in the study were similar that all have chief complaint of active ear discharge with a history of more than 3 months duration, a central perforation most often in antero inferior quadrant of tympanic membrane and a conductive hearing loss ranging from $30 \mathrm{~dB}$ SPL to $55 \mathrm{~dB}$ of SPL measured by pure tone audiometry.

The exclusion criteria are 1) a patient with attico-antral type of CSOM with attic or posterior marginal perforation, who may commonly have anaerobic bacterial pathogens, as our study did not use anaerobic culture media or 2) a patient who has been using an antibiotic within 7 days of clinical presentation.

Our institute has a policy of waiting for at least 4 weeks of discharge free interval before posting a patient with tubo-tympanic type of CSOM for tympanoplasty to avoid surgery in an inflamed middle ear environment. In case of attico-antral CSOM we do not wait for the discharge to subside before posting the patient to undergo ear surgery as complications can occur even during seemingly discharge free interval or the discharge may never subside. For this reason we have not included patients with attico-antral type of CSOM in the study.

From each patient of active CSOM, two swabs of the ear discharge are collected under aseptic precautions without surface contamination and are transported to Microbiology department. One swab was utilized for Gram's staining and the other was inoculated on Nutrient agar, Blood agar and MacConkey agar for bacterial culture. After overnight incubation at $37^{\circ} \mathrm{C}$ the culture plates were observed for growth. Single colony was stained by Gram's Method from each culture plate. Hanging drop was done if Gram negative bacilli were seen. The cultured bacteria are subjected to various biochemical tests depending on the organism like catalase test, oxidase test, urea hydrolysis test, phenol red test etc., at CAIMS microbiology lab (Figure 1). The organism isolated was tested for antibiotic sensitivity on Mueller-Hinton agar by Kirby-Bauer disc diffusion method according to National Committee for Clinical Laboratory Standards (NCCLS) criteria [2].

\section{Results}

In our study out of 90 patients 52 were males and 38 were females with in age range of 2 to 70 years. Out of total 100 samples collected from 90 patients suffering from tubo-tympanic CSOM in our study, 62 samples were culture positive. There was no statistically significant sex or age predilection for a sample to become cul-

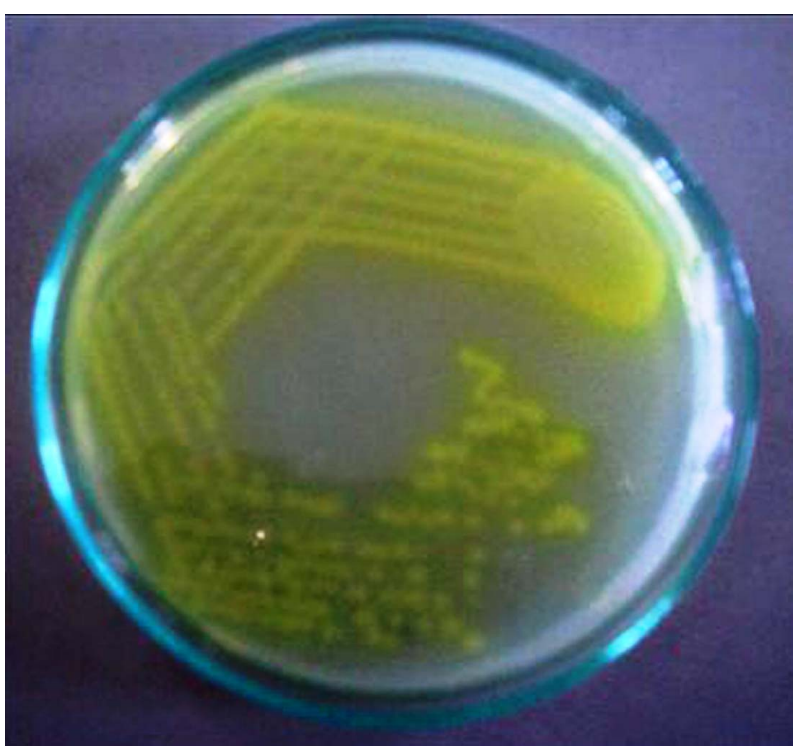

Figure 1. Fluorescin pigment (greenish yellow) produced by Pseudomonas aeruginosa on nutrient agar.

ture positive in our study. Among the 10 patients who had bilateral ear discharge 3 patients had same organism infecting both ears and two had different bacteria affecting each ear.

Among the 62 bacterial isolates, Pseudomonas aeruginosa was the most common bacterium cultured in 33 (54\%) samples, followed by Staphylococcus aureus in 7 (11.3\%) as indicated in Table 1.

Among the 33 isolates of Pseudomonas aeruginosa it was sensitive to Imipenem (94\%), Ciprofloxacin (85\%), Ceftazidime (82\%), and resistant to Cefepime (66\%), Piperacillin/Tazobactam (64\%), Cefoperazone (64\%), Cefotaxime (60\%), Amikacin (55\%), and others (Table 2 and Chart 1).

Staphylococcus aureus (Table 3) was sensitive to Ciprofloxacin (85\%), Gentamicin (72\%), Ofloxacin (72\%), Amoxicillin (57\%), Linezolid (57\%), and resistant to Cotrimoxazole (85\%), Amikacin (72\%), Cefotaxime (72\%), and Erythromycin (57\%).

Gram negative bacteria (Table 4) other than Pseudomonas aeruginosa were mostly sensitive to Ciprofloxacin (93\%) and resistant to Tetracycline (81\%), Ofloxacin (62.5\%), and Cefotaxime (44\%), Cefoperazone (37.5\%).

Staphylococcus saprophyticus was mostly sensitive to Ciprofloxacin (85\%) and Beta hemolytic Streptococci were sensitive to Bacitracin.

\section{Discussion}

Among 100 samples collected from 90 patients, 62 samples were bacterial culture positive with a culture positivity of $69 \%$. In studies done by Vikas Khanna et al. [3], V. K. Poorey et al. [4], Tanmoy Dev et al. [5], and S. Ni- 
Table 1. List of organisms isolated.

\begin{tabular}{ccc}
\hline Name of bacteria & $\begin{array}{c}\text { Number of } \\
\text { isolates }\end{array}$ & Percentage \\
\hline GRAM NEGATIVE BACTERIA & & \\
Pseudomonas aeruginosa & 33 & $54 \%$ \\
Proteus mirabilis & 5 & $8.06 \%$ \\
Klebsiella pneumoniae & 5 & $8.06 \%$ \\
Proteus vulgaris & 4 & $6.45 \%$ \\
Escherichia coli & 2 & $3.25 \%$ \\
GRAM POSITIVE COCCI & & \\
Staphylococcus aureus & 7 & $11.3 \%$ \\
Staphylococcus saprophyticus & 5 & $8.06 \%$ \\
Beta haemolytic Streptococci & 1 & $1.6 \%$ \\
\hline
\end{tabular}

Table 2. Antibiotic susceptibility pattern in Pseudomonas aeruginosa.

\begin{tabular}{cc}
\hline SENSITIVE & RESISTANT \\
\hline Imipenem $-31(94 \%)$ & Cefepime $-22(66 \%)$ \\
Ciprofloxacin - 28 (85\%) & Cefoperazone - 21 (64\%) \\
Ceftazidime $-27(82 \%)$ & Carbenicillin - 20 (60\%) \\
Levofloxacin $-23(70 \%)$ & Cefotaxime - $20(60 \%)$ \\
& Amikacin - $18(55 \%)$ \\
\hline
\end{tabular}

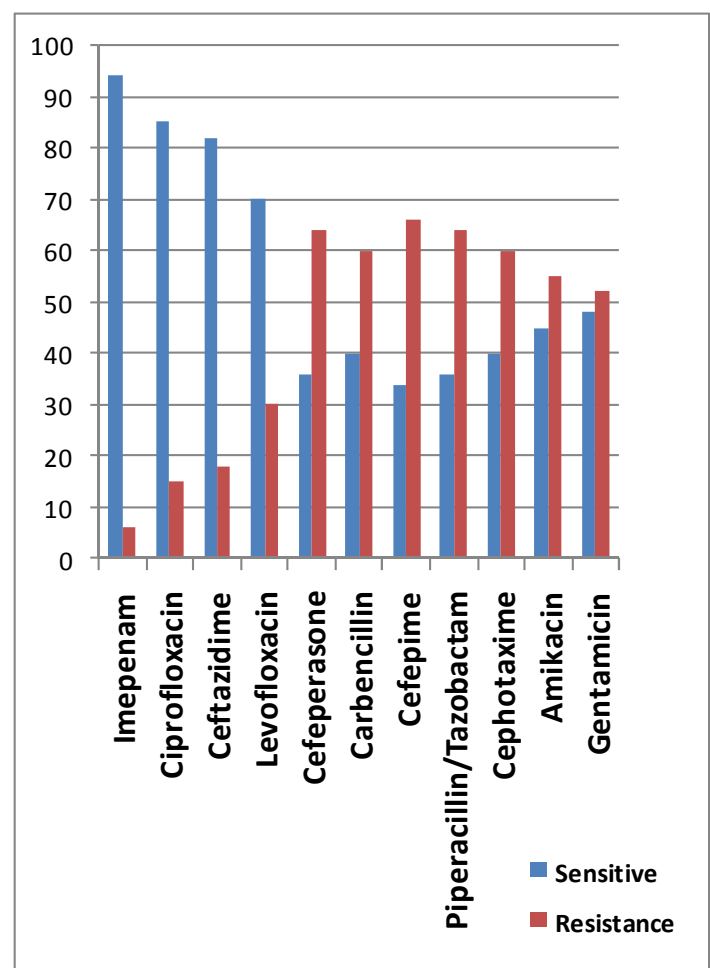

Chart 1. Antibiotic susceptibility pattern in Pseudomonas aeruginosa.
Table 3. Antibiotic susceptibility pattern in Staphylococcus aureus.

\begin{tabular}{cc}
\hline SENSITIVE & RESISTANT \\
\hline Ciprofloxacin $-6(85 \%)$ & Cotrimoxazole $-6(85 \%)$ \\
Gentamicin - 5 (72\%) & Amikacin - 5 (72\%) \\
Ofloxacin - 5 (72\%) & Cefotaxime - 5 (72\%) \\
Amoxicillin - 4 (57\%) & Erythromycin - 4 (57\%) \\
\hline
\end{tabular}

Table 4. Antibiotic susceptibility pattern in gram negative bacteria other than Pseudomonas aeruginosa.

\begin{tabular}{cc}
\hline SENSITIVE & RESISTANT \\
\hline Ciprofloxacin - 15 (93\%) & \\
Gentamicin - 13 (81\%) & Tetracycline - $13(81 \%)$ \\
Amoxycillin + Clavulanic acid - 12 (75\%) & Ofloxacin - $10(62.5 \%)$ \\
Ceftazidime - 11 (68.75\%) & Cefotaxime - 7 (44\%) \\
Amikacin - $10(62.5 \%)$ & Cefoperazone (37.5\%). \\
Levofloxacin - 8 (50\%) & \\
Piperacillin/Tazobactam - 8 (50\%) & \\
\hline
\end{tabular}

kakhlagh et al. [6] the culture positivity was $84 \%, 92 \%$, $53 \%$ and $82 \%$ respectively. In our study all were monobacterial cultures where as in studies done by Vikas Khanna et al. [3], V. K. Poorey et al. [4] polymicrobial or mixed cultures were obtained in $39 \%$ and $10 \%$ respectively.

Out of 62 bacterial isolates in the present study Pseudomonas aeruginosa was the predominant bacterium in 33 (54\%) followed by Staphylococcus aureus in 7 (11.3\%) isolates. Kenna et al. [7] found that Pseudomonas was the predominant organism (67\%) in their study. In study done by Vikas Khanna et al. [3] the most common bacterial isolate was Pseudomonas aeruginosa (40.57\%), followed by Staphylococcus aureus in 36.23\% of cases. S. Nikakhlagh et al. [6] studied that Staphylococcus aureus is common isolate in $32.4 \%$ followed by $21.69 \%$ of Pseudomonas aeruginosa. V. K. Poorey et al. [4] observed that Pseudomonas pyocyaneous was the most common organism isolated in $35.2 \%$, followed by Klebsiella aerogenes in $25.4 \%$. The observations made from different studies indicate there can be variation in causative organism based on ethnic, geographic factors.

In the present study Ciprofloxacin drug has emerged as the most effective antibiotic useful for the patients in our study which is sensitive against more than $85 \%$ of Pseudomonas, Staphylococci and other pathogens. In studies done by Sharma K. et al. [8] and V. K. Poorey et al. [4] Amikacin was the most effective drug. In our study the strains of Pseudomonas which were resistant to Ciprofloxacin were sensitive to Imipenem which is a similar finding observed by Jang et al. [9]. The mecha- 
nism of resistance is believed to be mediated by formation of biofilms by infecting organisms [10,11].

Out of 90 patients in our study more than 70 patients have used antibiotics during some part of their long duration of CSOM before presenting to us. Their drug history included most commonly Cefixime, Amoxicillin with Clavulanic acid and topical Ofloxacin which were not useful for them. After obtaining the culture sensitivity report they were put on oral and topical Ciprofloxacin and we have observed early response as most of the discharging ears were dry by 2 weeks of treatment. Those patients who were culture negative were successfully treated of their ear discharge by regular dry mopping of ear, a medical treatment with oral Ciprofloxacin and Ornidazole combination along with topical antifungal Clotrimazole ear drops. This observation is suggestive of a role of anaerobes and fungal pathogens in CSOM [12-14]. All the patients were offered tympanoplasty surgery 4 weeks after obtaining dry ear.

After the present study we now initially treat our tubotympanic CSOM patients with oral Ciprofloxacin empirically. Only those patients who fail to respond even after 2 weeks of ciprofloxacin treatment are subjected to culture and sensitivity test of their ear discharge. This approach of empirical treatment with initial ciprofloxacin has enabled us to successfully shorten the course of ear discharge and avoided the cost and time involved in sending each patient's ear discharge for microbiological study. We are making efforts to communicate the results of our study with otolaryngologists and physicians in our area for better patient care in our locality.

\section{Conclusion}

Our study, in the light of other various studies, indicates that there can be a variation in the organisms infecting and their susceptibility pattern based on season, locality and ethnicity of a patient with CSOM [15-17]. Hence it is prudent for any health centre catering to a defined area of population to conduct periodically the microbiological study of CSOM in order to install effective treatment protocols for the population.

\section{Acknowledgements}

We thank the faculty and staff of the department of Microbiology of CAIMS, Karimnagar, who helped us to carry out our study.

\section{REFERENCES}

[1] J. Acuin, "Chronic Suppurative Otitis Media, Burden of Illness and Management Options,” WHO Publications, 2004.

[2] National Committee for Clinical Laboratory Standards (NCCLS), "Performance Standards for Antimicrobial Disc
Susceptibility Test," 6th Edition, Approved Standard Wayne, PA, NCCLS, 1997, NCCLS Document $\mathrm{M}_{2}-\mathrm{A}_{6}$.

[3] V. Khanna, J. Chander, N. M. Nagarkar and A. Dass, "Clinicomicrobiologic Evaluation of Active Tubotympanic Type Chronic Suppurative Otitis Media,” Journal of Otolaryngology, Vol. 29, No. 3, 2000, pp. 148-153.

[4] V. K. Poorey and A. Iyer, "Study of Bacterial flora in CSOM and Its Clinical Significance," Indian Journal of Otolaryngology and Head \& Neck Surgery, Vol. 54, No. 2, 2002, pp. 91-95.

[5] T. Deb and D. Ray, "A Study of the Bacteriological Profile of Chronic Suppurative Otitis Media in Agartala,” Indian Journal of Otolaryngology and Head \& Neck Surgery, Vol. 64, No. 4, 2012, pp. 326-329. http://dx.doi.org/10.1007/s12070-011-0323-6

[6] S. Nikakhlagh, A. D. Khosravi, A. Fazlipour, M. Safarzadeh and N. Rashidi, "Microbiologic Findings in Patients with Chronic Suppurative Otitis Media,” Journal of Medical Sciences, Vol. 8, 2008, pp. 503-506.

http://dx.doi.org/10.3923/jms.2008.503.506

[7] M. A. Kenna, C. D. Bluestone, J. S. Reilly and R. P. Lusk, "Medical Management of Chronic Suppurative Otitis Media without Cholesteatoma in Children," Laryngoscope, Vol. 96, No. 2, 1986, pp. 146-151. http://dx.doi.org/10.1288/00005537-198602000-00004

[8] K. Sharma, A. Aggarwal and P. M. S. Khurana, "Comparison of Bacteriology in Bilaterally Discharging Ears in Chronic Suppurative Otitis Media," Indian Journal of Otolaryngology and Head \& Neck Surgery, Vol. 62, No. 2, 2010, pp. 153-157. http://dx.doi.org/10.1007/s12070-010-0021-9

[9] C. H. Jang and S. Y. Park, "Emergence of CiprofloxacinResistant Pseudomonas in Chronic Suppurative Otitis Media," Clinical Otolaryngology and Allied Sciences, Vol. 29, No. 4, 2004, pp. 321-323.

http://dx.doi.org/10.1111/j.1365-2273.2004.00835.x

[10] J. E. Dohar, P. A. Hebda, R. Veeh, M. Awad, et al., "Mucosal Biofilm Formation on Middle-Ear Mucosa in a Nonhuman Primate Model of Chronic Suppurative Otitis Media,” Laryngoscope, Vol. 115, No. 8, 2005, pp. 14691472.

http://dx.doi.org/10.1097/01.mlg.0000172036.82897.d4

[11] S. J. Daniel, "Topical Treatment of Chronic Suppurative Otitis Media," Current Infectious Disease Reports, Vol. 14, No. 2, 2012, pp. 121-127. http://dx.doi.org/10.1007/s11908-012-0246-8

[12] I. Brook, "The Role of Anaerobic Bacteria in Chronic Suppurative Otitis Media in Children: Implications for Medical Therapy,” Anaerobe, Vol. 14, No. 6, 2008, pp. 297-300.

http://dx.doi.org/10.1016/j.anaerobe.2008.12.002

[13] A. O. Ibekwe, Z. Al Shareef and A. Benayam, "Anaerobes and Fungi in Chronic Suppurative Otitis Media," The Annals of Otology, Rhinology, and Laryngology, Vol. 106, No. 8, 1997, pp. 649-652.

[14] P. Talwar, A. Chakrabarti, P. Kaur, et al., "Fungal Infection of Ear with Special Reference to Chronic Suppurative Otitis Media,” Mycopathologia, Vol. 104, No. 1, 1988, pp. 47-50. http://dx.doi.org/10.1007/BF00437923 
[15] A. Yildirim, H. Erdem, S. Kilic, S. Yetiser and A. Pahsa, "Effect of Climate on the Bacteriology of Chronic Suppurative Otitis Media,” The Annals of Otology, Rhinology, and Laryngology, Vol. 114, No. 8, 2005, pp. 652-655.

[16] A. H. Loy, A. L. Tan and P. K. Lu, "Microbiology of Chronic Suppurative Otitis Media in Singapore,” Singapore
Medical Journal, Vol. 43, No. 6, 2002, pp. 296-299

[17] R. S. Greval and S. Ram, "Bacteriological Patterns of Chronic Suppurative Otitis Media in Ludhiana," Indian Journal of Medical Sciences, Vol. 50, No. 6, 1996, pp. 192-195. 